\title{
Dust extinction and absorption: the challenge of porous grains
}

\author{
N. V. Voshchinnikov ${ }^{1,2}$, V. B. Il'in ${ }^{1,2}$, Th. Henning ${ }^{3}$, and D. N. Dubkova ${ }^{1}$ \\ 1 Sobolev Astronomical Institute, St. Petersburg University, Universitetskii prosp. 28, St. Petersburg, 198504 Russia \\ e-mail: nvv@astro.spbu.ru \\ 2 Isaac Newton Institute of Chile, St. Petersburg Branch, Russia \\ 3 Max-Planck-Institut für Astronomie, Königstuhl 17, 69117 Heidelberg, Germany
}

Received 5 May 2005 / Accepted 9 August 2005

\section{ABSTRACT}

In many models of dusty objects in space the grains are assumed to be composite or fluffy. However, the computation of the optical properties of such particles is still a very difficult problem. We analyze how the increase of grain porosity influences basic features of cosmic dust interstellar extinction, dust temperature, infrared bands and millimeter opacity.

It is found that an increase of porosity leads to an increase of extinction cross sections at some wavelengths and a decrease at others depending on the grain model. However, this behaviour is sufficient to reproduce the extinction curve in the direction of the star $\sigma$ Sco using current solar abundances. In the case of the star $\zeta$ Oph our model requires larger amounts of carbon and iron in the dust-phase than is available. Porous grains can reproduce the flat extinction across the 3-8 $\mu \mathrm{m}$ wavelength range measured for several lines of sight by ISO and Spitzer.

Porous grains are generally cooler than compact grains. At the same time, the temperature of very porous grains becomes slightly larger in the case of the EMT-Mie calculations in comparison with the results found from the layered-sphere model. The layered-sphere model predicts a broadening of infrared bands and a shift of the peak position to larger wavelengths as porosity grows. In the case of the EMT-Mie model variations of the feature profile are less significant. It is also shown that the millimeter mass absorption coefficients grow as porosity increases with a faster growth occurring for particles with Rayleigh/non-Rayleigh inclusions. As a result, for very porous particles the coefficients given by two models can differ by a factor of about 3 .

Key words. scattering - ISM: dust, extinction - comets: general - interplanetary medium - stars: individual: $\zeta$ Oph, $\sigma$ Sco - Galaxy: center

\section{Introduction}

The steady decrease of estimates of metal abundances in the solar atmosphere over the last years (Holweger 2001; Lodders 2003; Asplund et al. 2005) is a serious challenge not only to solar physics, but also to dust modelling. This calls for new dust models able to produce the same extinction with a smaller amount of solid material. A solution to the problem could be provided by an "admixture of vacuum" i.e. by the porosity of interstellar grains.

Grain aggregates with large voids can form during the growth of interstellar grains due to their coagulation in dense molecular cloud cores (Dorschner \& Henning 1995). The internal structure of such composite grains can be very complicated, but their optical properties are often calculated using the Mie theory for homogeneous spheres with an average refractive index derived from effective medium theory (EMT; see, e.g., Mathis \& Whiffen 1989; Jones 1988; Ossenkopf 1991; Mathis 1996; Li \& Greenberg 1998; Il'in \& Krivova 2000).

Another approach to calculate the optical properties of such aggregates is the application of complex, computationally time consuming methods such as the discrete dipole approximation
(DDA; see, e.g., Wright 1987; Kozasa et al. 1992, 1993; Wolff et al. 1994; Stognienko et al. 1995; Kimura \& Mann 1998).

Using the DDA, Voshchinnikov et al. (2005) examined the ability of the EMT-Mie approach to treat porous particles of different structure. They show that the latter approach can give relatively accurate results only if the very porous particles have small (in comparison with the wavelength of incident radiation) "Rayleigh" inclusions. Otherwise, the approach becomes inaccurate when the porosity exceeds $\sim 0.5$. At the same time, the optical properties of heterogeneous spherical particles having inclusions of various sizes (Rayleigh and non-Rayleigh) and very large porosity were found to closely resemble those of spheres with a large number $(\gtrsim 15-20)$ of different layers. The errors in extinction efficiency factors are smaller than 10-20\% if the size parameter $\lesssim 15$ and porosity is equal to 0.9 . Note that this consideration was restricted by spheres, not very absorbing materials (silicate and amorphous carbon) and the integral scattering characteristics (extinction, scattering, absorption efficiency factors, albedo and asymmetry parameter) but not the differential cross sections or elements of the scattering matrix. Nevertheless, very simple computational models instead 
of time-consuming DDA calculations give us a useful way to treat composite grains of different structure.

In this paper, we apply the particle models of porous interstellar dust grains based on the EMT-Mie and layeredsphere calculations. The models described in Sect. 2 are assumed to represent composite particles with small (Rayleigh) inclusions and inclusions of different sizes (Rayleigh and non-Rayleigh). The wavelength dependence of extinction is discussed in Sect. 3.1. Sections 3.2 and 3.3 contain the application of the models to calculations of the extinction curves in the directions of two stars, using new solar abundances and the near-infrared (IR) extinction in the directions along the Galactic plane. The next sections deal with grain temperatures (Sect. 4.1), profiles of IR silicate bands (Sect. 4.2), and grain opacities at $\lambda=1 \mathrm{~mm}$ (Sect. 4.3). These quantities are especially important for the analysis of observations of protoplanetary discs (Henning et al. 2005). Concluding remarks are presented in Sect. 5.

\section{Particle models}

Information about the structure of grains can be included in light scattering calculations directly (layered spheres) or can be used to find the optical constants (EMT-Mie model). We consider models of both types.

Following previous papers (Voshchinnikov \& Mathis 1999; Voshchinnikov et al. 2005), we construct layered grains as particles consisting of many concentric spherical layers of various materials, each with a specified volume fraction $V_{i}$ $\left(\Sigma_{i} V_{i} / V_{\text {total }}=1\right)$. Vacuum can be one of the materials, so a composite particle may have a central cavity or voids in the form of concentric layers. The number of layers is taken to be 18 since Voshchinnikov et al. (2005) have shown that this was enough to preclude an influence of the order of materials on the results. For a larger number of layers, one can speak of the optical characteristics determined by the volume fractions of different constituents only.

In the case of the EMT-Mie model, an average (effective) refractive index is calculated using the popular rule of Bruggeman (see, e.g., Chýlek et al. 2000; Krügel 2003). In this case, the average dielectric permittivity $\varepsilon_{\mathrm{eff}}{ }^{1}$ is calculated from

$\sum_{i} f_{i} \frac{\varepsilon_{i}-\varepsilon_{\mathrm{eff}}}{\varepsilon_{i}+2 \varepsilon_{\mathrm{eff}}}=0$

where $f_{i}=V_{i} / V_{\text {total }}$ is the volume fraction of the $i$ th material with the permittivity $\varepsilon_{i}$.

The amount of vacuum in a particle can be characterized by its porosity $\mathcal{P}(0 \leq \mathcal{P}<1)$

$\mathcal{P}=V_{\text {vac }} / V_{\text {total }}=1-V_{\text {solid }} / V_{\text {total }}$.

To compare the optical properties of porous and compact particles, one should consider the porous particles of radius (or size parameter)

$r_{\text {porous }}=\frac{r_{\text {compact }}}{(1-\mathcal{P})^{1 / 3}}=\frac{r_{\text {compact }}}{\left(V_{\text {solid }} / V_{\text {total }}\right)^{1 / 3}}$.

1 The dielectric permittivity is related to the refractive index as $\varepsilon=m^{2}$.
As "basic" constituents, we choose amorphous carbon (AC1; Rouleau \& Martin 1991) and astronomical silicate (astrosil; Laor \& Draine 1993). The refractive indices for these materials and some others considered in Sect. 3 were taken from the Jena-Petersburg Database of Optical Constants (JPDOC) described by Henning et al. (1999) and Jäger et al. (2003).

The application of the standard EMT is known to correspond to the case of particles having small randomly located inclusions of different materials (Bohren \& Huffman 1983). The optical properties of such particles have been well studied (see, e.g., Voshchinnikov 2004, and references therein).

However, one needs the DDA or other computationally time consuming techniques to treat particles with inclusions larger than the wavelength of incident radiation. The difference in the optical characteristics of particles with small and large inclusions has been discussed in previous studies (e.g., Wolff et al. 1994, 1998). The fact that this difference drastically grows with the porosity $\mathcal{P}$ and becomes quite essential already for $\mathcal{P} \gtrsim 0.5$ has been discovered only recently by Voshchinnikov et al. (2005). They also found that the scattering properties of particles with inclusions of different sizes (including those with sizes comparable to the wavelength) were very close to those of layered particles, having the same size and material volume fractions. A similar conclusion was reached for an ensemble of particles where each particle has inclusions of one (but different) size only. In both cases of "internal" or "external" mixing, the model of layered spheres can be applied.

These results are of particular importance for applications including the astronomical ones where we deal with very porous particles. Note that instead of time consuming calculations with DDA-like codes, one can use the exact solution to the light scattering problem for layered spheres, which is as quick as Mie theory, to estimate the properties of particles with Rayleigh/non-Rayleigh inclusions.

Thus, the models of the homogeneous sphere (with EMT) and layered spheres, both having fast implementations, allow one to probe the difference in optical properties caused by the different structure of scatterers.

\section{Interstellar extinction and interstellar abundances}

\subsection{Wavelength dependence of extinction}

As it is well known, the wavelength dependence of interstellar extinction $A(\lambda)$ is determined by the wavelength dependence of the extinction efficiencies $Q_{\text {ext }}(\lambda)$. This quantity is shown in Fig. 1 for particles of the same mass, but different porosity. The volume fractions of $\mathrm{AC} 1$ and astrosil are equal, i.e. $V_{\mathrm{AC} 1} / V_{\text {total }}=V_{\text {astrosil }} / V_{\text {total }}=1 / 2\left(V_{\text {solid }} / V_{\text {total }}\right)=1 / 2(1-\mathcal{P})$. The radius of compact grains is $r_{\mathrm{s} \text {, compact }}=0.1 \mu \mathrm{m}$. The dependence of $Q_{\text {ext }}$ on $\lambda$ for compact particles is close to that of the average interstellar extinction curve in the visible-near UV $\left(1 \mu \mathrm{m}^{-1} \leq \lambda^{-1} \leq 3 \mu \mathrm{m}^{-1}\right)$ where it can be approximated by the power law $A(\lambda) \propto \lambda^{-1.33}$ (see discussion in Voshchinnikov 2004). From Fig. 1 one can conclude that the extinction produced by particles with small inclusions and layers differs little for compact and slightly porous particles. 

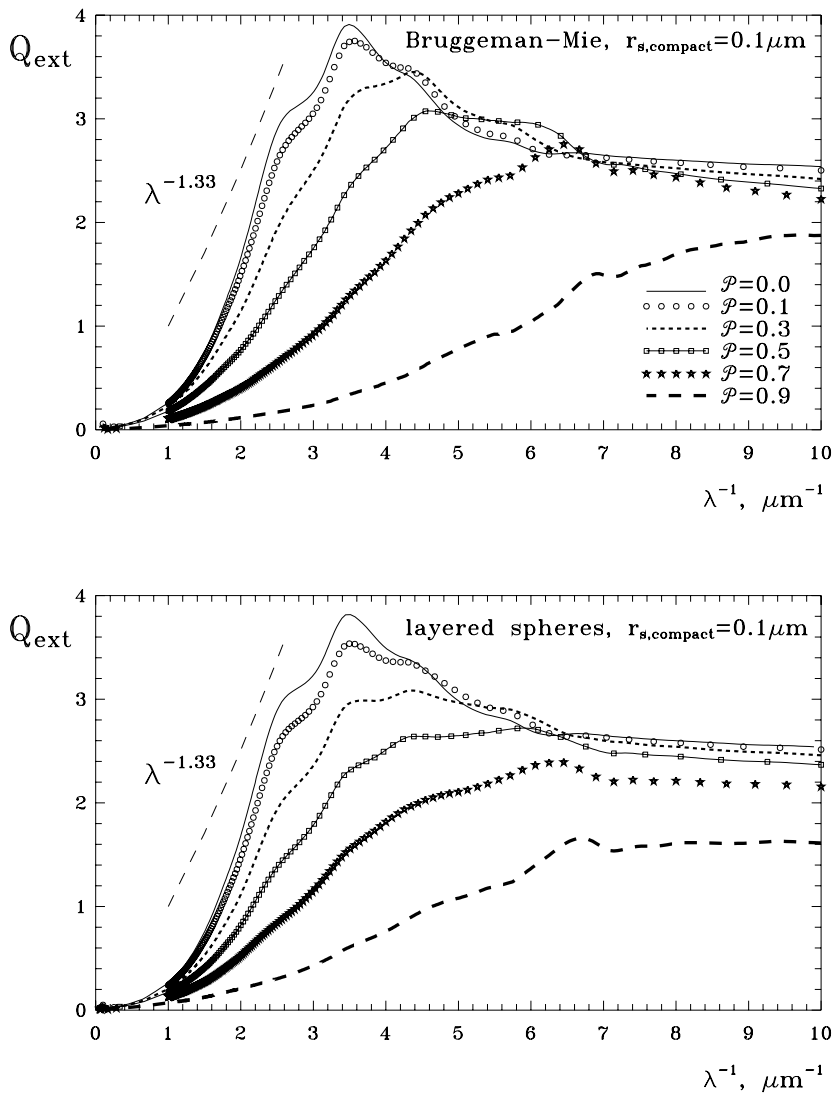

Fig. 1. Wavelength dependence of the extinction efficiency factor for spherical particles with $r_{\mathrm{s} \text {, compact }}=0.1 \mu \mathrm{m}$. The particles are of the same mass but of different porosity. Upper panel: calculations based on the EMT-Mie theory. Lower panel: calculations based on the layered-sphere theory.

The difference becomes most pronounced in the near and far$\mathrm{UV}$ (for $\lambda^{-1} \gtrsim 2.5-3 \mu \mathrm{m}^{-1}$ ).

As follows from Fig. 1, the wavelength dependence of extinction flattens as porosity increases. It is well known (see, e.g., Greenberg 1978) that different particles produce comparable extinction if the products of their size $r$ and refractive index are close, i.e.

$r|m-1| \approx$ const.

The average refractive index of particles with a larger fraction of vacuum is closer to 1 . Despite a larger radius (e.g., from Eq. (3) follows that $r_{\mathrm{s}}=0.22 \mu \mathrm{m}$ if $\mathcal{P}=0.9$ and $r_{\mathrm{s} \text {, compact }}=$ $0.1 \mu \mathrm{m}$ ), the product given by Eq. (4) decreases because of an drop of $|m-1|$. This implies that a steeper extinction with the wavelength dependence closer to $\propto \lambda^{-1.33}$ is produced by for compact particles with larger radii and, consequently, a larger amount of solid material. This behaviour is also observed for particles of other masses (i.e., compact spheres of other radii). Therefore, an interpretation of the observed interstellar extinction curve using only very porous grains should not give any gain in dust-phase abundances and would contradict the wavelength behaviour.
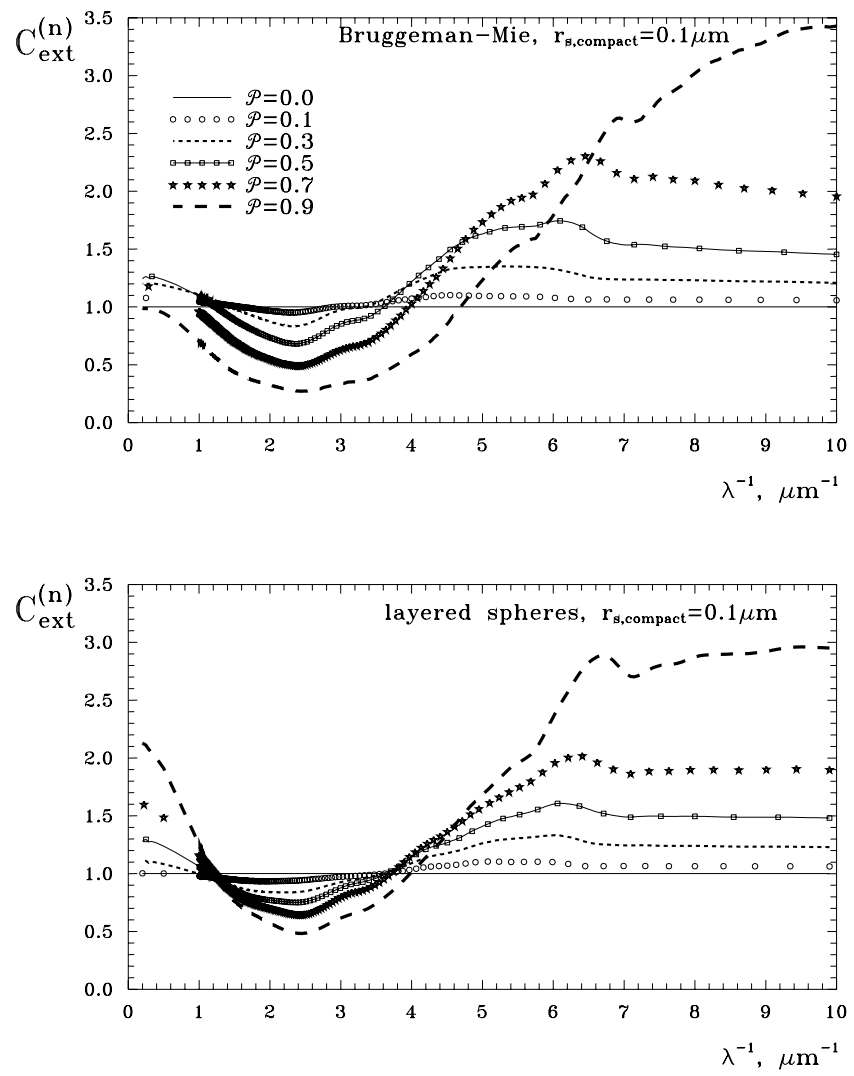

Fig. 2. The same as in Fig. 1 but now for normalized extinction cross section.

The role of porosity in extinction is better seen from Fig. 2 where we give the wavelength dependence of the normalized cross section

$$
\begin{aligned}
C_{\mathrm{ext}}^{(\mathrm{n})} & =\frac{C_{\text {ext }}(\text { porous grain })}{C_{\mathrm{ext}}(\text { compact grain of same mass })} \\
& =(1-\mathcal{P})^{-2 / 3} \frac{Q_{\text {ext }}(\text { porous grain })}{Q_{\text {ext }}(\text { compact grain of same mass })} .
\end{aligned}
$$

This quantity shows how porosity influences the extinction cross section. As follows from Fig. 2, both models predict a growth of extinction of porous particles in the far-UV and a decrease in the visual-near-UV part with growing $\mathcal{P}$. However, the wavelength interval where $C_{\mathrm{ext}}^{(\mathrm{n})}<1$ is narrower and the minimum is less deep in the case of layered spheres. In comparison with compact grains and particles with Rayleigh inclusions, particles with Rayleigh/non-Rayleigh inclusions can also produce rather large extinction in the near-IR part of spectrum. This is especially important for the explanation of the flat extinction across the 3-8 $\mu$ m wavelength range measured for several lines of sight (see Sect. 3.3). At the same time, as follows from Fig. 3, for particles with $r_{\mathrm{s} \text {, compact }}>0.1 \mu \mathrm{m}$ the normalized UV cross sections grow faster for the Bruggeman-Mie theory than for layered spheres. Thus, an addition of vacuum into particles does not mean an automatic growth of extinction at all wavelengths and a saving in terms of solid-phase elements. Evidently, the final decision can be made after fitting the theoretical calculations with observations at many wavelengths. 

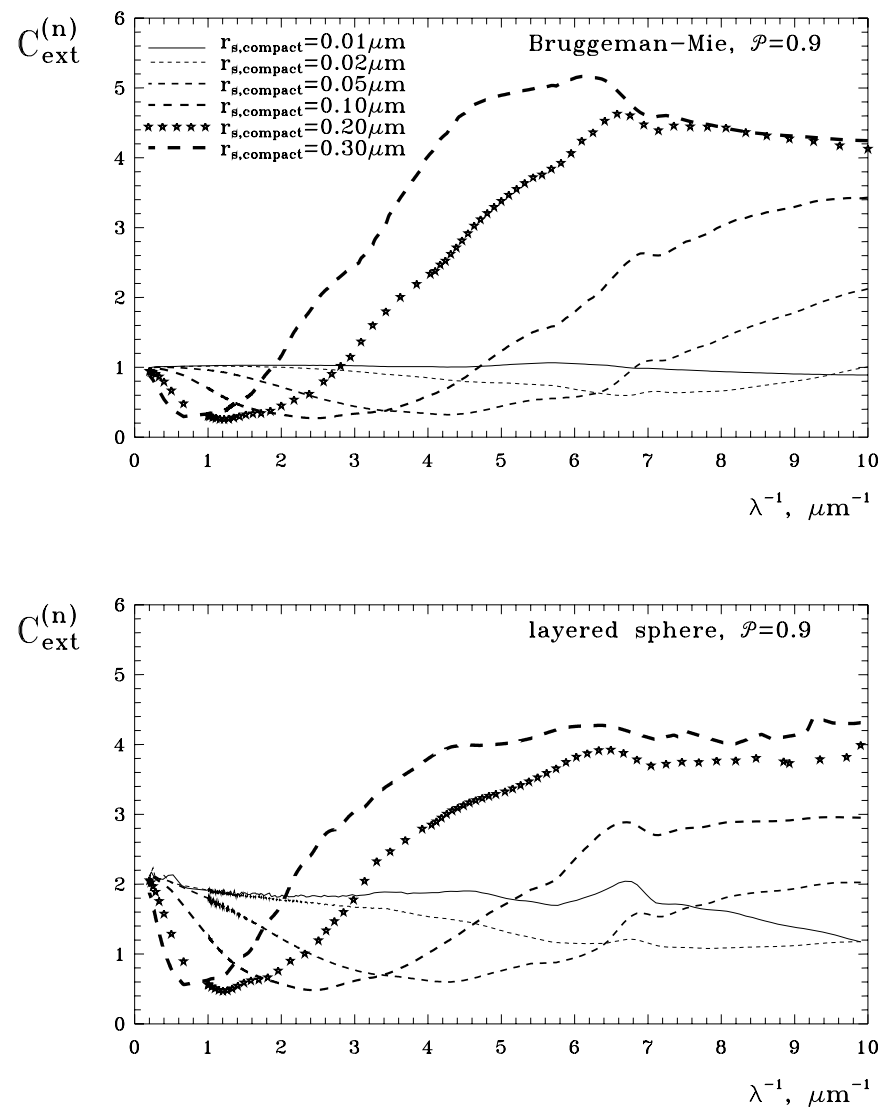

Fig. 3. Wavelength dependence of the normalized extinction cross section for spherical particles with the same porosity $\mathcal{P}=0.9$. Upper panel: calculations based on the EMT-Mie theory. Lower panel: calculations based on the layered-sphere theory.

\subsection{Extinction in the directions to the stars $\zeta$ Oph and $\sigma$ Sco}

The basic requirement for any model of interstellar dust is the explanation of the observed extinction law along with the dustphase abundances of elements in the interstellar medium. These abundances are obtained as the difference between the cosmic reference abundances and the observed gas-phase abundances. However, the cosmic abundances are not yet conclusively established and usually this causes a problem. For many years, the solar abundances were used as the reference ones, until the photospheres of the early-type stars were found not to be as rich in heavy elements as the solar photosphere was (Snow \& Witt 1996). These stellar abundances caused the so-called "carbon crisis". Abundances of the most important dust-forming elements $(\mathrm{C}, \mathrm{O}, \mathrm{Mg}, \mathrm{Si}, \mathrm{Fe})$ required by the dust models were larger than available. However, during the past several years the solar abundances dropped and now they approach the stellar ones (see Asplund et al. 2005). Evidently, some abundances determined by Sofia \& Meyer (2001) for F and G stars must be revised downward as it has been done recently for the Sun. This should lead to the agreement between abundances found for stars of different types. Note also that the current solar abundances of oxygen and iron (see Table 3 ) are close to those found from high-resolution X-ray spectroscopy. Juett et al. (2004) investigated the oxygen $\mathrm{K}$-shell interstellar absorption edge in seven X-ray binaries and evaluated the total $\mathrm{O}$ abundances. These abundances lie between $467 \mathrm{ppm}^{2}$ and $492 \mathrm{ppm}$. Schulz et al. (2002) evaluated the total abundance of iron towards the object Cyg X-1 to be $[\mathrm{Fe} / \mathrm{H}]_{\text {cosmic }} \approx 25 \mathrm{ppm}$.

We applied the model of multi-layered porous particles to explain the absolute extinction in the direction to the two stars. A first estimate has been made in order to find a possibility to enlarge the extinction per unit mass and to minimize the amount of solid phase material. Several materials as components of composite grains were considered. Among the carbonaceous species, the amorphous carbon in the form of $\mathrm{Be} 1$ (Rouleau \& Martin 1991) was found to produce the largest extinction. Also the extinction of iron oxides strongly increases with the growth of porosity. Although there are no very good constraints on the abundance of oxides, $\mathrm{FeO}$ is considered as a possible carrier of the $21 \mu \mathrm{m}$ emission observed in the spectra of post-AGB stars (Posch et al. 2004). Very likely, such particles particles can be produced in redox reactions (Duley 1980; Jones 1990).

We fitted the observed extinction toward the stars $\zeta$ Oph and $\sigma$ Sco. For these stars there exist well determined extinction curves and gas-phase abundances. It is also important that the major part of extinction in these directions is produced in one diffuse interstellar cloud (Savage \& Sembach 1996; Zubko et al. 1996). This allows us to exclude possible large variations in dust composition along the line of sight.

Observed and calculated extinction curves are plotted in Figs. 4 ( $\zeta$ Oph) and 5 ( $\sigma$ Sco). As follows from the previous Section, the use of only porous or only compact grains apparently does not result significant benefit in the solid-phase abundances. Therefore, our models are the combination of compact and porous particles. They consist of three or four grain populations:

(I). Porous composite (multi-layered) particles (Be1 - 5\%, pyroxene, $\mathrm{Fe}_{0.5} \mathrm{Mg}_{0.5} \mathrm{SiO}_{3}-5 \%$ for $\zeta$ Oph or forsterite, $\mathrm{Mg}_{2} \mathrm{SiO}_{4}-5 \%$ for $\sigma$ Sco and vacuum $-90 \%$ ) with the power-law size distribution having an exponential decay $n_{\mathrm{d}}\left(r_{\mathrm{s}}\right) \propto r_{\mathrm{s}}^{-2.5} \exp \left(-10 / r_{\mathrm{s}}\right)$. The lower/upper cutoff in the size distribution is $0.015 \mu \mathrm{m} / 0.25 \mu \mathrm{m}$ and $0.05 \mu \mathrm{m} / 0.50 \mu \mathrm{m}$ for $\zeta$ Oph and $\sigma$ Sco, respectively.

(II). Small compact graphite ${ }^{3}$ grains with a narrow power-law size distribution $\left(n_{\mathrm{d}}\left(r_{\mathrm{s}}\right) \propto r_{\mathrm{s}}^{-2.5}, r_{\mathrm{s}}=0.01-0.02 \mu \mathrm{m}\right)$.

(III). Porous composite grains of magnetite $\left(\mathrm{Fe}_{3} \mathrm{O}_{4}-2 \%\right.$, vacuum $-98 \%$ for $\zeta \mathrm{Oph}$ and $\mathrm{Fe}_{3} \mathrm{O}_{4}-8 \%$, vacuum $-92 \%$ for $\sigma$ Sco) with a power-law size distribution $n_{\mathrm{d}}\left(r_{\mathrm{s}}\right) \propto$ $r_{\mathrm{s}}^{-2.5}$. The lower/upper cut-off in the size distribution is $0.005 \mu \mathrm{m} / 0.25 \mu \mathrm{m}$ and $0.05 \mu \mathrm{m} / 0.35 \mu \mathrm{m}$ for $\zeta$ Oph and $\sigma$ Sco, respectively.

(IV). Compact grains of forsterite $\left(\mathrm{Mg}_{2} \mathrm{SiO}_{4}\right)$ with the powerlaw size distribution (only for $\zeta$ Oph, $n_{\mathrm{d}}\left(r_{\mathrm{s}}\right) \propto r_{\mathrm{s}}^{-3.5}$, $\left.r_{\mathrm{s}, \min }=0.10 \mu \mathrm{m}, r_{\mathrm{s}, \max }=0.25 \mu \mathrm{m}\right)$.

\footnotetext{
${ }^{2}$ Parts per million hydrogen atoms.

3 The calculations for graphite were made in the " $2 / 3-1 / 3$ " approximation: $Q_{\mathrm{ext}}=2 / 3 Q_{\mathrm{ext}}\left(\varepsilon_{\perp}\right)+1 / 3 Q_{\mathrm{ext}}\left(\varepsilon_{\|}\right)$, where $\varepsilon_{\perp}$ and $\varepsilon_{\|}$are the dielectric functions for two cases of orientation of the electric field relative to the basal plane of graphite.
} 


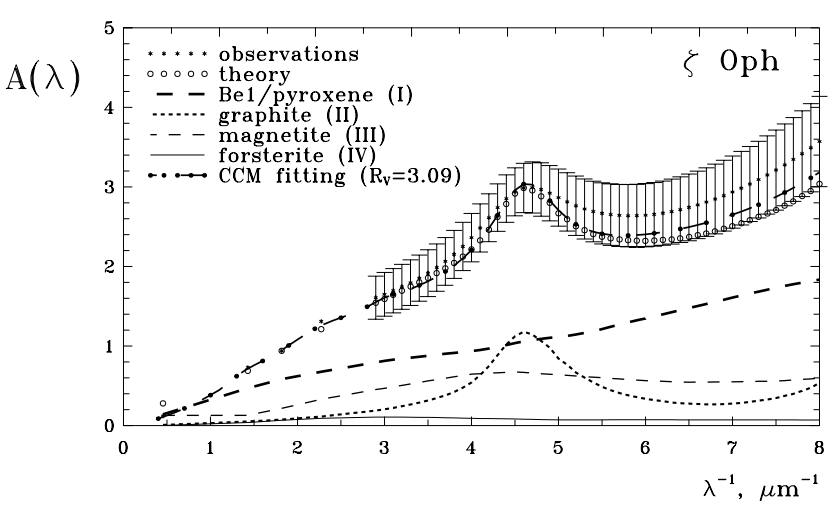

Fig. 4. Observed and calculated extinction in the direction to $\zeta$ Oph. The errors of the observations are the result of a parameterization of the observations (see Fitzpatrick \& Massa 1990). The contribution to the theoretical extinction from different components is also shown. The dot-dashed curve is the approximation with the observed value $R_{\mathrm{V}}$ as suggested by Cardelli et al. (1989).

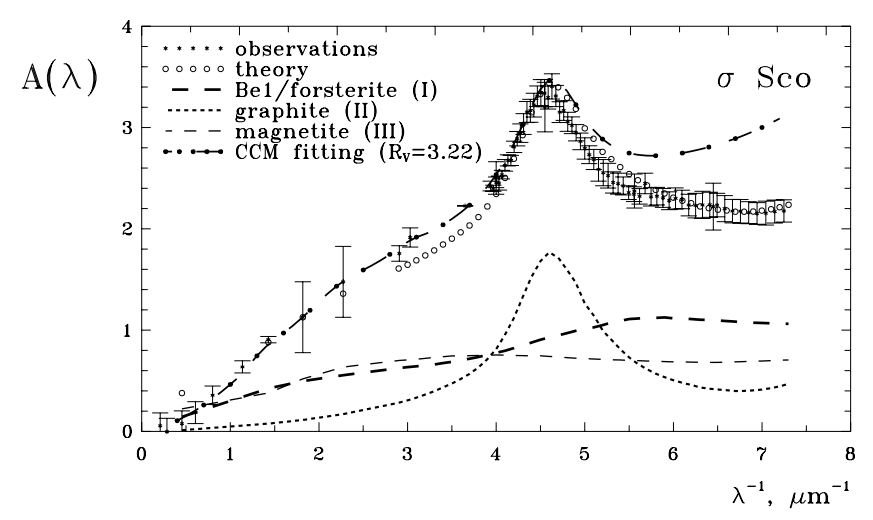

Fig. 5. The same as in Fig. 4 but now for $\sigma$ Sco. The observational data were taken from Wegner (2002).

Table 1. Contribution of different grain populations to $A_{\mathrm{V}}$ and dustphase abundances for the model of $\zeta$ Oph (in ppm).

\begin{tabular}{llccccc}
\hline \hline Component & $A_{\mathrm{V}}$ & $\mathrm{C}$ & $\mathrm{O}$ & $\mathrm{Mg}$ & $\mathrm{Si}$ & $\mathrm{Fe}$ \\
\hline (I) Be1/pyroxene/vacuum & 0.58 & 123 & 68 & 11.3 & 22.6 & 11.3 \\
(II) Graphite & 0.075 & 96 & & & & \\
(III) Magnetite/vacuum & 0.22 & & 33 & & & 24.8 \\
(IV) Forsterite & 0.065 & & 23 & 11.4 & 5.7 & \\
\hline Total & 0.94 & 219 & 124 & 22.7 & 28.2 & 36.1 \\
\hline
\end{tabular}

Figures 4 and 5 also contain the extinction curves calculated using the approximation suggested by Cardelli et al. (1989) with the coefficients revised by O'Donnell (1994). Cardelli et al. (1989) found that the extinction curves from the UV through the IR could be characterized as a one-parameter family dependent on the ratio of the total extinction to the selective one $R_{\mathrm{V}}=A_{\mathrm{V}} / E(\mathrm{~B}-\mathrm{V})$. We used the observed values of $R_{\mathrm{V}}$ in order to plot the CCM approximation. It is seen that this relation describes quite well the extinction for $\zeta$ Oph but not for $\sigma$ Sco.

The contributions from different components to the calculated extinction are given in Tables 1 and 2 and shown in Figs. 4 and 5. The Tables contain also the dust-phase
Table 2. Contribution of different grain populations to $A_{\mathrm{V}}$ and dustphase abundances for the model of $\sigma$ Sco (in ppm).

\begin{tabular}{llccccc}
\hline \hline Component & $A_{\mathrm{V}}$ & $\mathrm{C}$ & $\mathrm{O}$ & $\mathrm{Mg}$ & $\mathrm{Si}$ & $\mathrm{Fe}$ \\
\hline (I) Be1/forsterite/vacuum & $0 . \mathrm{m} 50$ & 58 & 35.4 & 17.7 & 8.8 & \\
(II) Graphite & 0.11 & 79 & & & & \\
(III) Magnetite/vacuum & 0.52 & & 35.4 & & & 26.6 \\
\hline Total & $1 . \mathrm{m} 13$ & 137 & 71 & 17.7 & 8.8 & 26.6 \\
\hline
\end{tabular}

Table 3. Observed and model dust-phase abundances (in ppm).

\begin{tabular}{cccccc}
\hline \hline \multirow{2}{*}{ Element } & Solar* & \multicolumn{2}{c}{$\zeta$ Oph } & \multicolumn{2}{c}{$\sigma$ Sco } \\
& abundance & obs & model & obs & model \\
\hline $\mathrm{C}$ & 245 & 110 & 219 & 176 & 137 \\
$\mathrm{O}$ & 457 & 126 & 124 & 85 & 71 \\
$\mathrm{Mg}^{* *}$ & 33.9 & 31.9 & 22.7 & 30.9 & 17.7 \\
$\mathrm{Si}$ & 34.2 & 32.6 & 28.2 & 32.4 & 8.8 \\
$\mathrm{Fe}$ & 28.2 & 28.2 & 36.1 & 27.9 & 26.6 \\
\hline
\end{tabular}

* According to Asplund et al. (2005).

** The abundance of $\mathrm{Mg}$ was recalculated with the oscillator strengths from Fitzpatrick (1997).

abundances of five dust-forming elements for several grain populations. They were calculated for ratios of the extinction cross-section to particle volume averaged over grain size distribution (see Eq. (3.36) in Voshchinnikov 2004).

Table 3 gives the current solar abundances of five dustforming elements according to Asplund et al. (2005) as well as the "observed" (solar minus gaseous) and model abundances.

The dust-phase abundances in the line of sight to the star $\zeta$ Oph (HD 149757) were taken from Table 2 of Snow \& Witt (1996). In our calculations, we adopted the following quantities for $\zeta$ Oph: a total extinction $A_{\mathrm{V}}=0.94^{4}$, colour excess $E(B-V)=0.32$ and total hydrogen column density $N(\mathrm{H})=1.35 \times 10^{21} \mathrm{~cm}^{-2}$ (Savage \& Sembach, 1996). The extinction curve was reproduced according to the parameterization of Fitzpatrick \& Massa (1990).

For $\sigma$ Sco (HD 147165), we used the extinction curve, the colour excess $E(B-V)=0.35$ and the total extinction $A_{\mathrm{V}}=1 \mathrm{~m}$. 13 according to Wegner (2002). The hydrogen column density $N(\mathrm{H})=2.46 \times 10^{21} \mathrm{~cm}^{-2}$ was adopted from Zubko et al. (1996). The gas-phase abundances were taken from Allen et al. (1990).

The dust-phase abundances required by the model are larger than the observed ones in the direction to $\zeta$ Oph (for $\mathrm{C}$ and $\mathrm{Fe}$ ) and smaller than the observed abundances in the direction to $\sigma$ Sco. Note that for $\sigma$ Sco the required amount of $\mathrm{C}$ and $\mathrm{Si}$ in dust grains is the lowest in comparison with previous modelling. This is due to the use of highly porous particles which give considerable extinction in the UV and nearIR (see Figs. 2 and 3) and allow one to "save" material. For example, the extinction model of $\sigma$ Sco with compact grains

\footnotetext{
4 This value was obtained from the relation $A_{\mathrm{V}}=1.12 E(V-K)$ (Voshchinnikov \& Il'in 1987) and a colour excess $E(V-K)=0$. 84 (Serkowski et al. 1975).
} 
presented by Zubko et al. (1996) requires 240-260 ppm of C and 20-30 ppm of Si and the model of Clayton et al. (2003) needs $155 \mathrm{ppm}$ of $\mathrm{C}$ and $36 \mathrm{ppm}$ of $\mathrm{Si}$ (cf. $137 \mathrm{ppm}$ and 8.8 ppm from Table 3).

The models presented above are based on the light scattering calculations for particles with Rayleigh/non-Rayleigh inclusions (layered spheres). It is evident that the observed extinction can be also reproduced if we use the particles with Rayleigh inclusions (i.e., if we apply the EMT-Mie theory). Our estimates show that despite a larger extinction in the UV this model requires more material in the solid phase in comparison with layered spheres because of a smaller extinction in the visual-near IR part of the spectrum.

\subsection{Near infrared extinction in the Galactic plane}

We now consider the possibility of explaining the flat extinction across the $3-8 \mu \mathrm{m}$ wavelength range observed for several lines of sight. This flattening was first measured by Lutz et al. (1996) toward the Galactic center with ISO, using hydrogen recombination lines. Later Lutz (1999) confirmed the effect using more recombination lines. Recently, Indebetouw et al. (2005) found a similar flat extinction along two lines of sight: $l=42^{\circ}$ and $l=284^{\circ}$. The extinction was obtained at seven wavelengths $(1.2-8 \mu \mathrm{m})$ by combining images from the Spitzer Space Telescope with the 2MASS point-source catalog.

We used the average extinction given in Table 1 of Indebetouw et al. (2005) and transformed it into magnitudes of extinction per kpc using the measured value $A_{\mathrm{K}} / D=0 \mathrm{~m} .15 \pm$ $0.1 \mathrm{kpc}^{-1}$ (Indebetouw et al. 2005). The observations are plotted in Fig. 6 together with three theoretical curves. Because we have little information about the UV-visual extinction and gas-phase abundances in these directions, we (rather arbitrarily) applied the model used for $\zeta$ Oph (porous component (I): Be1/pyroxene, porosity 90\%; see Sect. 3.2). This model (short dashed curve in Fig. 6) well explains the flat extinction at $\lambda>3 \mu \mathrm{m}^{5}$ but the extinction in the $J$ and $H$ bands is too small. Compact particles (long-dashed curve in Fig. 6) produce even larger extinction at these bands than the observed one. However, the extinction from such particles at longer wavelengths decreases rapidly. Our preliminary analysis shows that particles with a porosity of about 0.6 (solid curve in Fig. 6) can be chosen as an appropriate model. Evidently, a similar curve can be obtained as a combination of compact and very porous particles. Quite close extinction can be found with the CCM approximation and the standard value $R_{\mathrm{V}}=3.1$. We arbitrarily extrapolated this approximation to long wavelengths $\left(\lambda^{-1}<0.3 \mu \mathrm{m}^{-1}\right)$ where it gives too small extinction.

Extinction produced by porous grains was also rather flat between 1.0 and $2.2 \mu \mathrm{m}$ (for example, $A(\lambda) \propto \lambda^{-1.3}$ for $\mathcal{P}=$ $0.6)$ as was detected for several ultracompact HII regions with $A_{\mathrm{V}} \gtrsim 15^{\mathrm{m}}$ (Moore et al. 2005).

In order to calculate the dust-phase abundances for models presented in Fig. 6 we estimated the total hydrogen

\footnotetext{
${ }^{5}$ Note that porous particles from magnetite (component (III); see Sect. 3.2) cannot fit well the extinction at these wavelengths because of a bump at $\lambda \approx 2 \mu \mathrm{m}$.
}

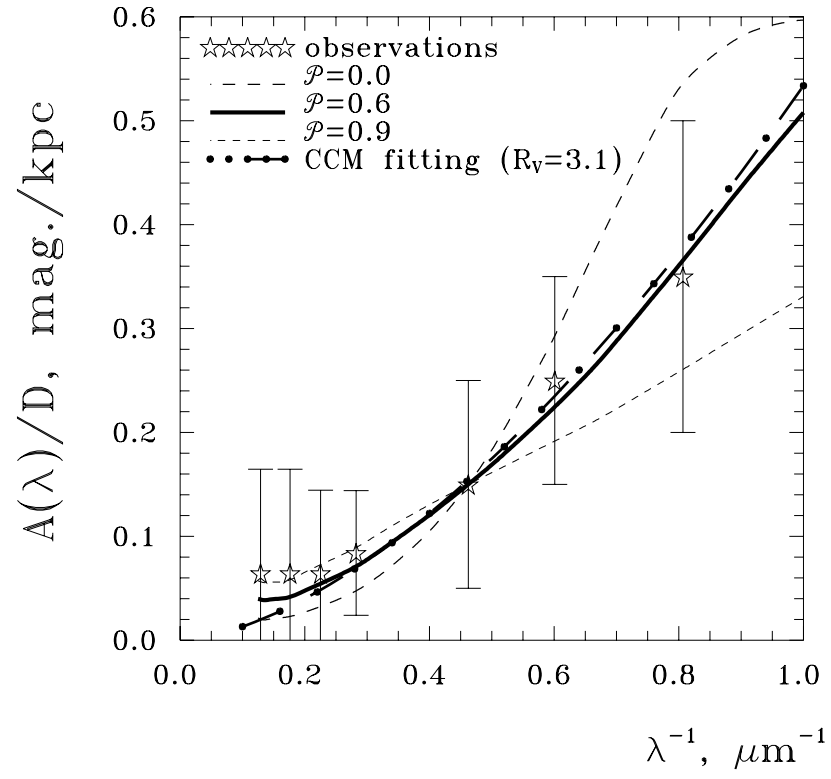

Fig. 6. Observed and calculated extinction in the near-IR part of spectrum. The observations correspond to the average extinction for two lines of sight along the Galactic plane (Indebetouw et al. 2005) transformed into magnitudes of extinction per kpc. The theoretical extinction was calculated for component (I) of the model used for $\zeta$ Oph ( $\mathcal{P}=0.9$, short dashed curve). Two other curves correspond to the same component but with another particle porosity. The dot-dashed curve is the approximation of Cardelli et al. (1989) with $R_{\mathrm{V}}=3.1$.

Table 4. Dust-phase abundances (in ppm) and visual extinction for three models presented in Fig. 6.

\begin{tabular}{cccc}
\hline \hline Element & $\mathcal{P}=0$ & $\mathcal{P}=0.6$ & $\mathcal{P}=0.9$ \\
\hline $\mathrm{C}$ & 96 & 84 & 62 \\
$\mathrm{O}$ & 53 & 46 & 34 \\
$\mathrm{Mg}$ & 8.9 & 7.7 & 5.7 \\
$\mathrm{Si}$ & 18 & 16 & 11 \\
$\mathrm{Fe}$ & 8.9 & 7.7 & 5.7 \\
\hline$A_{\mathrm{V}}$ & 0.60 & 0.79 & 0.61 \\
\hline
\end{tabular}

column density using Eqs. (3.26), (3.27) and (3.22) from Voshchinnikov (2004). First, we found the column density of atomic hydrogen $N(\mathrm{HI})$ from the extinction at $\mathrm{J}$ band and then transformed $N(\mathrm{HI})$ into a total hydrogen column density $N(\mathrm{H})$ using the ratio of total to selective extinction $R_{\mathrm{V}}=3.1$. A value of $N(\mathrm{H}) / D=2.79 \times 10^{21} \mathrm{~cm}^{-2} \mathrm{kpc}^{-1}$ was obtained. The calculated abundances are given in Table 4 , which also contains the visual extinction calculated for three models. Note that the model of grains with porosity $\mathcal{P}=0.6$ gives the largest contribution to $A_{\mathrm{V}}$ in comparison with two other models.

There are useful observational data for foreground stars in the field $l=284^{\circ}$. For stars HD 90273, HD 93205 and HD 93222, Wegner (2002) and Barbaro et al. (2004) estimate the values of $R_{\mathrm{V}}$ which lie between 3.4 and 4.0. For HD 90273 Barbaro et al. (2004) also find an anomalously high gas to dust ratio $N(\mathrm{H}) / E(B-V)=1.04 \times 10^{22}$ atoms cm$~^{-2} \mathrm{mag}^{-1}$. Enlargement of $R_{\mathrm{V}}$ increases the required dust-phase 

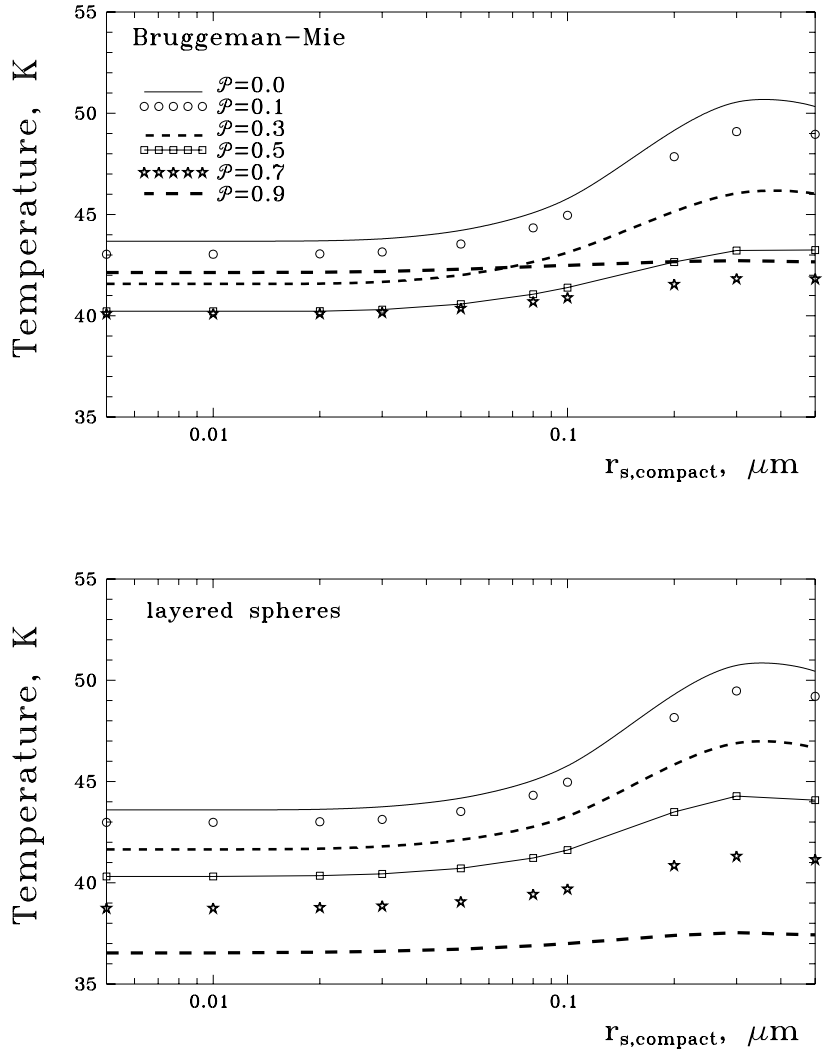

Fig. 7. Size dependence of the temperature for spherical particles. The particles are located at a distance of $10^{4} R_{\star}$ from a star with an effective temperature $T_{\star}=2500 \mathrm{~K}$. Upper panel: calculations based on the EMT-Mie theory. Lower panel: calculations based on the layeredsphere theory.

abundances while the decrease of the gas to dust ratio reduces them. André et al. (2003) measured the interstellar gas-phase oxygen abundances along the sight lines toward 5 early-type stars with $l=285^{\circ} .3-287^{\circ} .7$ and $b=-5^{\circ} .5+0^{\circ} .1$. The values of $[\mathrm{O} / \mathrm{H}]_{\mathrm{g}}$ vary from $356 \mathrm{ppm}$ to $512 \mathrm{ppm}$, the average value being $443 \mathrm{ppm}$. This gives for the mean dust-phase abundance $[\mathrm{O} / \mathrm{H}]_{\mathrm{d}}=14 \mathrm{ppm}$. However, the extinction curves for HD 93205 and HD 93222 published by Wegner (2002) have strong UV bumps and flat extinction in the far-UV. This means that extinction can be mainly produced by carbonaceous grains. Evidently, the most reasonable way to solve the problem of abundances is a re-examination of the reference cosmic abnudances and a detailed study of their local values.

\section{Infrared radiation}

\subsection{Dust temperature}

The commonly used equilibrium temperature of cosmic grains is derived from a balance between the energy gain due to absorption of the UV and visual stellar photons and the energy loss due to re-emission of IR photons. The temperature of porous and compact particles of different size and porosity is shown in Figs. 7 and 8 as a function of particle size and porosity, respectively. The results were calculated for particles located at a distance of $10^{4} R_{\star}$ from a star with an effective

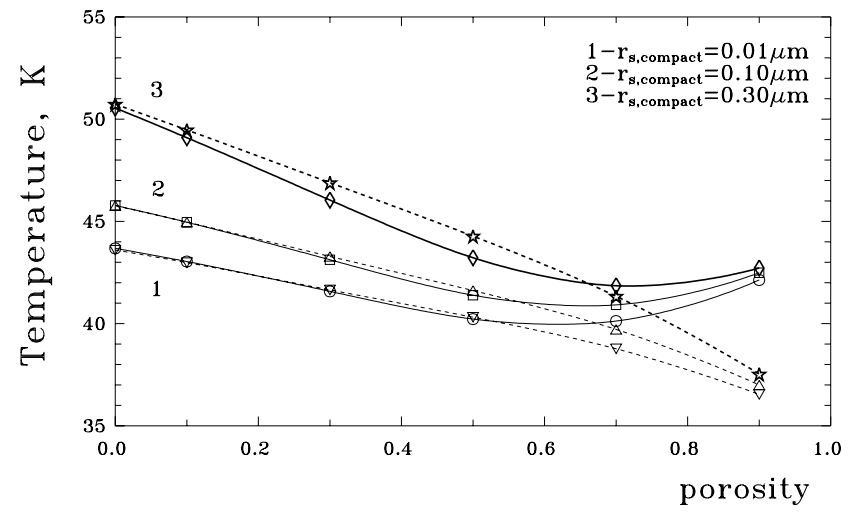

Fig. 8. Dependence of the dust temperature on particle porosity. Solid lines: calculations based on the EMT-Mie theory. dashed lines: calculations based on the layered-sphere theory. Other parameters are the same as in Fig. 7.

temperature $T_{\star}=2500 \mathrm{~K}$. In the case of the layered spheres, an increase of the vacuum fraction causes a decrease of the grain temperature if the amount of the solid material is kept constant. This behaviour holds for particles of all sizes as well as for particles located closer to the star or farther away and for other values of $T_{\star}$. If the EMT-Mie theory is applied, the temperature drops when the porosity grows up to $\sim 0.7$ and then starts to increase (see Fig. 7, upper panel and Fig. 8). Such a behaviour corresponds to the results of Greenberg \& Hage (1991) who found an increase of temperature for large grain porosity (see Fig. 4 in their paper).

As it follows from Figs. 7 and 8, the difference in the temperature of very porous grains calculated using the two models can reach $\sim 6 \mathrm{~K}$ or $\sim 15 \%$ while the temperature of compact $(\mathcal{P}=0)$ composite grains differs by less than $1 \%$. Note that the relative difference in temperatures of $\sim 15 \%$ between particles of the two types is kept for other stellar temperatures (e.g., in the case of the Sun or an interstellar radiation field).

The intermediate porosity of grains leads to a shift of the peak position of IR emission to larger wavelengths in comparison with compact particles. This occurs independently of particle structure (small or various size inclusions). However, very porous grains with Rayleigh/non-Rayleigh inclusions are expected to be systematically cooler than particles with Rayleigh inclusions. Such a difference can be of great importance at a lower temperature regime because it can influence the growth/destruction of mantles on grains in molecular clouds.

\subsection{Infrared features}

It is well known that the shape of the IR dust features is a good indicator of the particle size and chemical composition. With an increase of the size, a feature becomes wider and eventually fades away. For example, in the case of compact spherical grains of astrosil, the $10 \mu \mathrm{m}$ and $18 \mu \mathrm{m}$ features disappear when the grain radius exceeds $\sim 2-3 \mu \mathrm{m}$. Observed differences in small scale structure of the features are usually attributed to variations of the composition (e.g., changes of the ratio of magnesium to iron in silicates) or material state (amorphous/crystalline). 

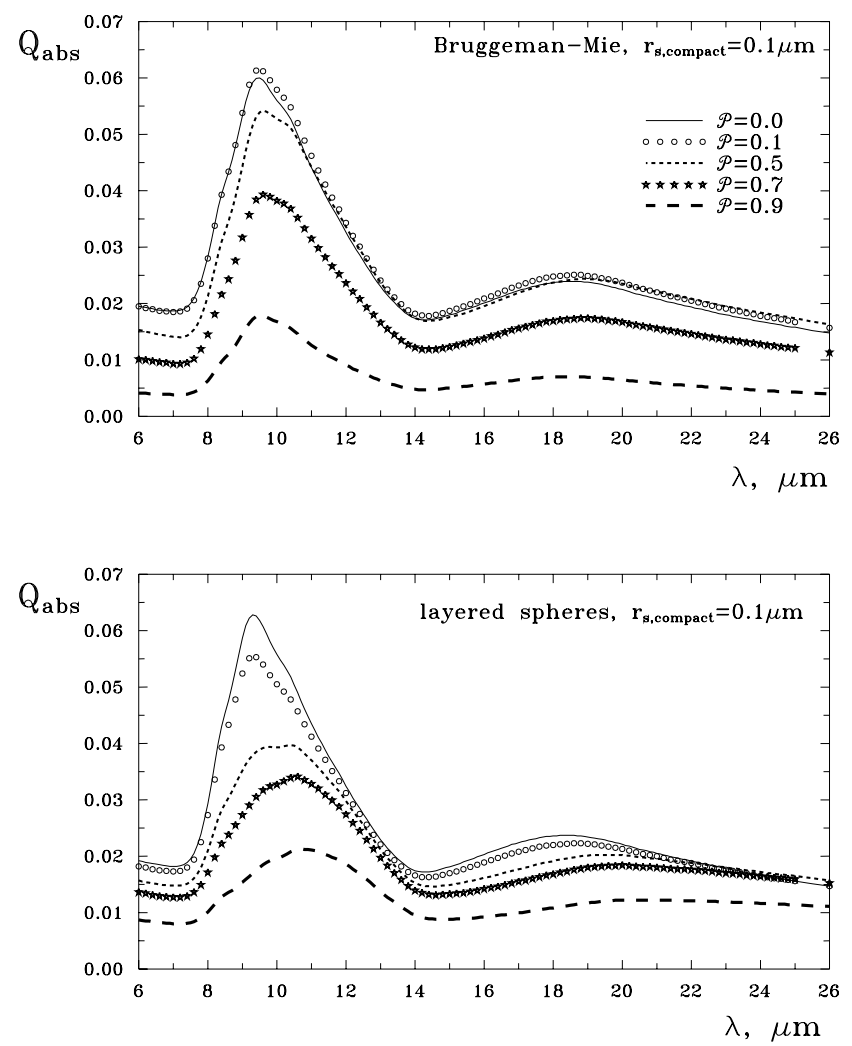

Fig. 9. Wavelength dependence of the absorption efficiency factors for spherical particles of radius $r_{\mathrm{s}, \text { compact }}=0.1 \mu \mathrm{m}$. Upper panel: calculations based on the EMT-Mie theory. Lower panel: calculations based on the layered-sphere theory.

In Fig. 9 we compare the wavelength dependence of the absorption efficiency factors for particles of the same mass but different structure. The upper panel shows results obtained with the EMT-Mie model for particles with Rayleigh inclusions. It can be seen that the central position and the width of the dust features does not really change. Larger changes occur for the layered-sphere model (Fig. 9, lower panel). In this case a growth of $\mathcal{P}$ causes a shift of the center of the feature to longer wavelengths and its broadening. For particles with $\mathcal{P}=0.9$, the $10 \mu \mathrm{m}$ feature transforms into a plateau while the $18 \mu \mathrm{m}$ feature disappears.

We plotted in Fig. 10 our data from Fig. 9 in a normalized manner together with observations of $\beta$ Pictoris made by Knacke et al. (1993) and Telesco \& Knacke (1991). As follows from Fig. 10, for given optical constants of the silicate the observed shape of the $10 \mu \mathrm{m}$ feature is better reproduced by either compact or porous particles with small size inclusions of materials. Note that in the case of $\beta$ Pictoris the EMT-Mie calculations were earlier used by Li \& Greenberg (1998) for the explanation of the $10 \mu \mathrm{m}$ emission feature and by Voshchinnikov \& Krügel (1999) for the interpretation of the positional and wavelength dependence of polarization. The best fit was obtained for very porous particles: $\mathcal{P} \approx 0.95$ and 0.76 , respectively.

Figure 11 shows the normalized absorption efficiency factors for spherical particles of radius $r_{\mathrm{s} \text {, compact }}=2 \mu \mathrm{m}$. For compact grains the $10 \mu \mathrm{m}$ feature almost disappears. When the porosity increases the strength of the feature grows in the case
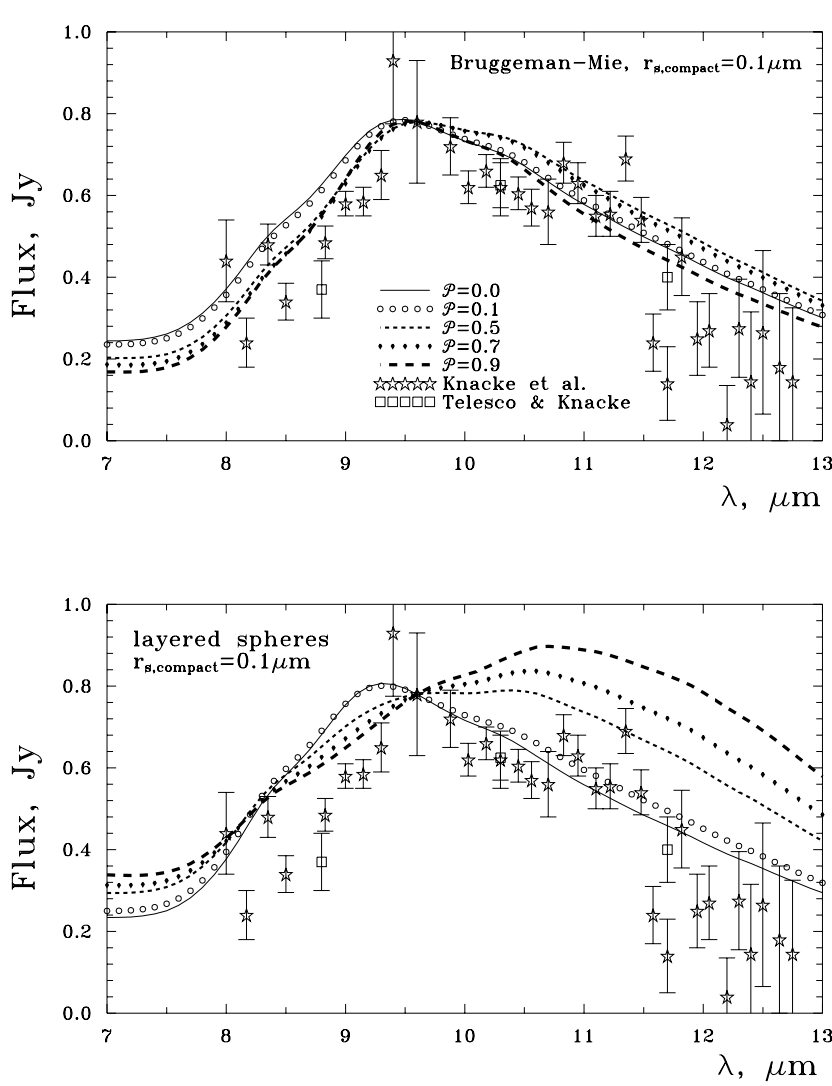

Fig. 10. Emission in the disc around the star $\beta$ Pictoris in the region of silicate $10 \mu \mathrm{m}$ band. Stars and squares are the observations of Knacke et al. (1993) and of Telesco \& Knacke (1991). The curves present the results of calculations for particles of radius $r_{\mathrm{s}, \text { compact }}=0.1 \mu \mathrm{m}$ as shown in Fig. 9 but normalized at $\lambda=9.6 \mu \mathrm{m}$.

of the Bruggeman-Mie calculations. This tendency coincides with the results shown in Fig. 7 of Hage \& Greenberg (1990) who found that the higher the porosity, the sharper the silicate emission became. For the case of layered spheres the feature becomes only slightly stronger but its peak shifts to longer wavelengths.

A standard "compact" approach to the modelling of the $10 \mu \mathrm{m}$ feature was used by van Boekel et al. (2003, 2005) and Przygodda et al. (2003) who considered the flattening of the $10 \mu \mathrm{m}$ feature as an evidence of grain growth in the discs around Herbig Ae/Be stars and T Tauri stars, respectively. Our investigations show that the variations of the shape of the feature and its position and strength can also be attributed to the change of porosity and relative amount of carbon in composite grains of small sizes.

\subsection{Dust opacities}

The dust opacity or the mass absorption coefficient of a grain material $\kappa(\lambda)$ enters directly in the expression for the dust mass of an object $M_{\mathrm{d}}$ which is determined from optically thin millimeter emission

$M_{\mathrm{d}}=\frac{F_{\mathrm{mm}}(\lambda) D^{2}}{\kappa(\lambda) B_{\lambda}\left(T_{\mathrm{d}}\right)}$ 

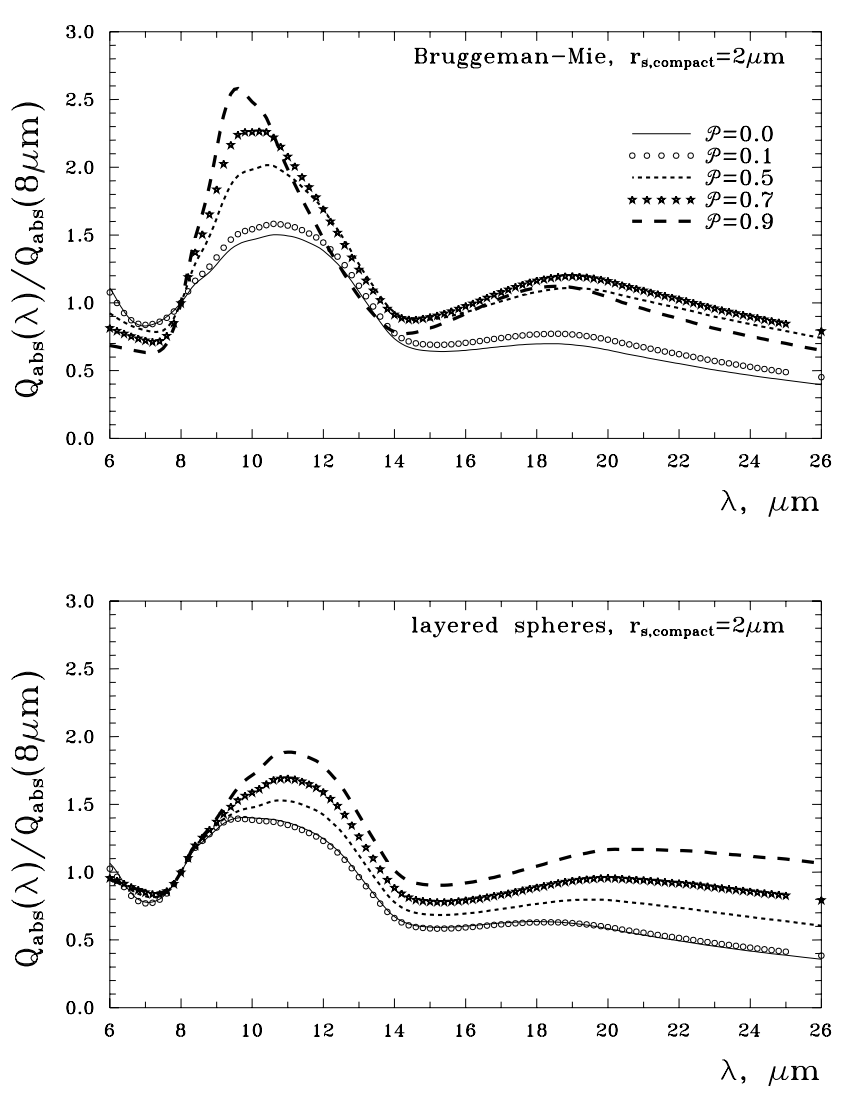

Fig. 11. Wavelength dependence of the normalized absorption efficiency factors for spherical particles of radius $r_{\mathrm{s} \text {, compact }}=2 \mu \mathrm{m}$. Upper panel: calculations based on the EMT-Mie theory. Lower panel: calculations based on the layered-sphere theory.

Here, $F_{\mathrm{mm}}(\lambda)$ is the observed flux, $D$ the distance to the object, $B_{\lambda}\left(T_{\mathrm{d}}\right)$ the Planck function, $T_{\mathrm{d}}$ the dust temperature. The mass absorption coefficient $K(\lambda)$ depends on the particle volume $V_{\text {total }}$, the material density $\rho_{\mathrm{d}}$ and the extinction cross-section $C_{\text {ext }}$ as follows:

$\kappa(\lambda)=\frac{C_{\mathrm{ext}}}{\rho_{\mathrm{d}} V_{\mathrm{total}}} \approx \frac{3}{\rho_{\mathrm{d}}}\left(\frac{2 \pi}{\lambda}\right) \operatorname{Im}\left\{\frac{\varepsilon_{\mathrm{eff}}-1}{\varepsilon_{\mathrm{eff}}+2}\right\}$.

At long wavelengths the scattering can be neglected $\left(C_{\text {ext }} \approx\right.$ $C_{\text {abs }}$ ) and $C_{\text {abs }}$ can be evaluated in the Rayleigh approximation. Then the mass absorption coefficient does not depend on the particle size as shown in the right part of Eq. (7). The effective dielectric permittivity $\varepsilon_{\mathrm{eff}}$ in Eq. (7) can be found from the Bruggeman rule (see Eq. (1)) or the layered-sphere rule of the EMT (see Eqs. (7), (8) in Voshchinnikov et al. 2005).

Extensive studies of the mass absorption coefficient dependence on the material properties and grain shape are summarized by Henning (1996) who, in particular, notes that the opacities at $1 \mathrm{~mm}$ are considerably larger for non-spherical particles than for spheres (see also Ossenkopf \& Henning 1994). We find that a similar effect (an increase of opacity in comparison with compact spheres) is produced by inclusion of a large fraction of vacuum into the particles. This follows from Table 5 where the opacities at $\lambda=1 \mathrm{~mm}$ are presented. This table contains the results for particles consisting of three materials (AC1, astrosil and vacuum) or two materials (AC1 or astrosil and vacuum).
In the first case, the volume fractions of $\mathrm{AC} 1$ and astrosil are equal $\left(V_{\mathrm{AC} 1} / V_{\text {total }}=V_{\text {astrosil }} / V_{\text {total }}=1 / 2(1-\mathcal{P})\right)$ while in the second case the volume fraction of solid material is $1-\mathcal{P}$. It can be seen that the values of $\kappa$ are generally larger for particles with a larger fraction of vacuum. This is related to the decrease of the particle density $\rho_{\mathrm{d}}$ which is calculated as the volume-average quantity. As the mass of dust in an object is proportional to $\rho_{\mathrm{d}}$ (see Eqs. (6) and (7)), the assumption of porous grains can lead to considerably smaller mass estimates. Note that the opacities are larger for more absorbing carbon particles. A similar effect was noted by Quinten et al. (2002) who theoretically studied the wavelength dependence of extinction of different carbonaceous particles. They also showed that the far IR extinction was larger for clusters of spheres and spheroids than for compact spheres. A very large enhancement of the submm opacities was found by Ossenkopf $\&$ Henning (1994) in the case of pure carbon aggregates or carbon on silicate grains.

Using Eq. (6) and data from Table 5 shows how the particle porosity and structure can influence estimates of dust mass in an object. The mass ratio estimates can be found in the Rayleigh-Jeans approximation

$\frac{M_{\mathrm{d}}(\text { compact })}{M_{\mathrm{d}}(\text { porous })}=\frac{\kappa_{\text {porous }}(\lambda) T_{\mathrm{d}, \text { porous }}}{\kappa_{\text {compact }}(\lambda) T_{\mathrm{d}, \text { compact }}}$.

With grain temperatures from Fig. 8 and the values of $\kappa$ for composite grains and EMT-Mie theory (the third column in Table 5) we can find that the ratio $M_{\mathrm{d}}$ (compact) $/ M_{\mathrm{d}}(\mathcal{P}=0.9)$ lies between $\sim 1.3$ and $\sim 1.5$. If the layered-sphere model is used (the forth column in Table 5) the ratio increases to 3.8-4.3. This means that the calculated mass of an object can be reduced if compact grains are replaced by porous ones.

The ratio of dust masses calculated for two grain models is

$$
\frac{M_{\mathrm{d}}(\text { EMT-Mie })}{M_{\mathrm{d}}(\text { layered sphere })}=\frac{\kappa_{\text {lay sphere }}(\lambda) T_{\mathrm{d} \text {,lay sphere }}}{\kappa_{\mathrm{EMT}-\mathrm{Mie}}(\lambda) T_{\mathrm{d}, \mathrm{EMT}-\text { Mie }}} \approx 2.8 .
$$

The numerical value was obtained for particles consisting of $\mathrm{AC} 1$, astrosil and vacuum with $\mathcal{P}=0.9$ and the temperature ratio $T_{\text {d,lay sphere }} / T_{\text {d,EMT-Mie }}=0.85$ as discussed in Sect. 4.1. If we consider particles of the same porosity but consisting of two materials, the ratio of masses will be even larger (3.1 for AC1 and 4.3 for astrosil). Thus, one can overestimate the mass of an object by a factor of 3 or more if the EMT-Mie model is applied, since real dust grains in molecular cloud cores should be very porous and should have non-Rayleigh inclusions. Another case when the effect can be important is in circumstellar discs, e.g. Takeushi et al. (2005) used $\kappa=0.3 \mathrm{~cm}^{2} / \mathrm{g}$ at $\lambda=1 \mathrm{~mm}$ for highly porous silicate grains, which is a good approximation only for particles with small size inclusions (see Table 5).

\section{Concluding remarks}

We have considered how the porosity of composite cosmic dust grains can affect their optical properties important for interpretation of observations of interstellar, circumstellar and cometary dust. Two models of particle structure were used. Particles of the first kind had well-mixed inclusions small in 
Table 5. Mass absorption coefficients at $\lambda=1 \mathrm{~mm}$ of compact and porous spheres consisting of $\mathrm{AC} 1^{*}$ and (or) astrosil ${ }^{* *}$.

\begin{tabular}{|c|c|c|c|c|c|c|c|c|c|}
\hline \multirow{3}{*}{$\mathcal{P}$} & \multicolumn{3}{|c|}{$\mathrm{AC} 1+$ astrosil } & \multicolumn{3}{|c|}{ Astrosil } & \multicolumn{3}{|c|}{$\mathrm{AC} 1$} \\
\hline & \multirow[t]{2}{*}{$\rho_{\mathrm{d}}$} & \multicolumn{2}{|c|}{$\kappa, \mathrm{cm}^{2} / \mathrm{g}$} & \multirow[t]{2}{*}{$\rho_{\mathrm{d}}$} & \multicolumn{2}{|c|}{$\kappa, \mathrm{cm}^{2} / \mathrm{g}$} & \multirow[t]{2}{*}{$\rho_{\mathrm{d}}$} & \multicolumn{2}{|c|}{$\kappa, \mathrm{cm}^{2} / \mathrm{g}$} \\
\hline & & Brugg.-Mie & lay. spheres & & Brugg.-Mie & lay. spheres & & Brugg.-Mie & lay. spheres \\
\hline 0.00 & 2.58 & 1.58 & 1.58 & 3.30 & 0.310 & 0.310 & 1.85 & 4.37 & 4.37 \\
\hline 0.10 & 2.32 & 1.89 & 1.55 & 2.97 & 0.371 & 0.334 & 1.66 & 5.13 & 4.60 \\
\hline 0.30 & 1.80 & 2.75 & 1.87 & 2.31 & 0.548 & 0.446 & 1.30 & 7.09 & 5.77 \\
\hline 0.50 & 1.29 & 3.83 & 2.57 & 1.65 & 0.778 & 0.646 & 0.925 & 9.22 & 7.88 \\
\hline 0.70 & 0.772 & 3.94 & 4.04 & 0.990 & 0.794 & 1.05 & 0.555 & 9.14 & 11.9 \\
\hline 0.90 & 0.258 & 2.45 & 8.12 & 0.330 & 0.431 & 2.20 & 0.185 & 5.94 & 21.8 \\
\hline
\end{tabular}

comparison with the wavelength, while those of the second kind consisted of very thin, cyclically repeating layers. Earlier we showed that the optical properties of such layered particles are close to those of particles with small and large inclusions (see Voshchinnikov et al. 2005). As effective medium theories give reliable results for particles with small inclusions, two very different particle structure models can be simply realized and extensive computations can be performed.

For both models, we studied how an increase of the volume fraction of vacuum could change the extinction efficiencies at different wavelengths, temperature of dust grains, profiles of the IR silicate bands and dust millimeter opacities. It is found that the models begin to differ essentially when the porosity exceeds $\sim 0.5$. This difference appears as lower temperatures (Sect. 4.1), shifted central peaks of the silicate bands (Sect. 4.2) and larger millimeter opacities (Sect. 4.3) for layered particle model in comparison with that based on EMT calculations. The latter model also requires larger dust-phase abundances than the layered model (Sect. 3.2) to produce the same interstellar extinction.

The assumption that interstellar particles have only small size inclusions looks to some extent artificial (excluding, of course, the case of special laboratory samples). Therefore, we believe that the layered sphere model well describing light scattering by very porous quasispherical particles with inclusions of different sizes should find wide applications in interpretation of different phenomena. In particular, this model has good perspectives to explanation of the flat interstellar extinction observed in the near-IR part of spectrum (Sect. 3.3) and variations of the shape of the silicate feature detected in spectra of T Tau and Herbig Ae/Be stars (the results will be in a subsequent next paper).

Acknowledgements. We are grateful to Walter Wegner for the possibility to use unpublished data and to Bruce Draine for comments on an earlier version of the paper. We are also grateful to the referee Michael Wolff and scientific editor Anthony Jones for useful suggestions. N.V.V. acknowledges the hospitality of the Max-PlanckInstitut für Astronomie where this work was finished. The work was partly supported by grant 1088.2003.2 of the President of the Russian Federation for leading scientific schools.

\section{References}

Allen, M. M., Snow, T. P., \& Jenkins, E. B. 1990, ApJ, 355, 130

André, M. K., Oliveira, C. M., Howk, J. C., et al. 2003, ApJ, 591, 1000 Asplund, M., Grevesse, N., \& Sauval, A. J. 2005, in Cosmic Abundances as Records of Stellar Evolution and Nucleosynthesis, ed. Th. G. Barnes, \& F. N. Bash, ASP Conf. Ser., 336, 25

Barbaro, G., Geminale, A., Mazzei, P., \& Congiu, E. 2004, MNRAS, 353, 760

Bohren, C. F., \& Huffman, D. R. 1983, Absorption and Scattering of Light by Small Particles (New York: J. Wiley \& Sons)

Cardelli, J. A., Clayton, G. C., \& Mathis, J. S. 1989, ApJ, 345, 245

Chýlek, P., Videen, G., Geldart, D. J. W., Dobbie, J. S., \& Tso, H. C. W. 2000, in Light Scattering by Nonspherical Particles, ed. M. I. Mishchenko et al. (San Francisco: Academic Press), 274

Clayton, G. C., Wolf, M. J, Sofia, U. J., Gordon, K. D., \& Misselt, K. A. 2003, ApJ, 588, 871

Dorschner, J., \& Henning, Th. 1995, A\&AR, 6, 271

Duley, W. W. 1980, ApJ, 240, 950

Fitzpatrick, E. L. 1997, ApJ, 482, L199

Fitzpatrick, E. L., \& Massa, D. L. 1990, ApJS, 72, 163

Greenberg, J. M. 1978, Interstellar Dust, in Cosmic Dust, ed. J. A. M. McDonnel, 187

Greenberg, J. M., \& Hage, J. L. 1991, in Chemistry in Space, ed. J. M. Greenberg, \& V. Pirronello (Kluwer), 363

Hage, J. I., \& Greenberg, J. M. 1990, ApJ, 361, 251

Henning, Th. 1996, in The Cosmic Dust Connection, ed. J. M. Greenberg (Kluwer), 399

Henning, Th., \& Stognienko, R. 1993, A\&A, 280, 609

Henning, Th., Il'in, V. B., Krivova, N. A., Michel, B., \& Voshchinnikov, N. V. 1999, A\&AS, 136, 405

Henning, Th., Dullemond, C. P., Dominik, C., \& Wolf, S. 2005, in Planet Formation: Observations, Experiments and Theory, ed. H. Klahr, \& W. Brandner (Cambridge: Cambridge University Press), in press

Holweger, H. 2001, in Solar and Galactic Composition, ed. R. F. Wimmer-Schweingruber (AIP), 23

Il'in, V. B., \& Krivova, N. A. 2000, Astron. Lett., 26, 379

Indebetouw, R., Mathis, J. S., Babler, B. L., et al. 2005, ApJ, 619, 931

Jäger, C., Il'in, V. B., Henning, Th., et al. 2003, JQSRT, 79, 765

Jones, A. P. 1988, MNRAS, 234, 209

Jones, A. P. 1990, MNRAS, 245, 331

Juett, A. M., Schulz, N. S., \& Chakrabarty, D. 2004, ApJ, 612, 308

Kimura, H., \& Mann, I. 1998, JQSRT, 60, 425

Knacke, R. F., Fajardo-Acosta, S. B., Telesco, C. M., et al. 1993, ApJ, 418,440 
Kozasa, T., Blum, J., \& Mukai, T. 1992, A\&A, 263, 423

Kozasa, T., Blum, J., Okamoto, H., \& Mukai, T. 1993, A\&A, 276, 278

Krügel, E. 2003, The Physics of Interstellar Dust (London: Institute of Physics Publishing)

Laor, A., \& Draine, B. T. 1993, ApJ, 402, 441

Li, A., \& Greenberg, J. M. 1998, A\&A, 331, 291

Lodders, K. 2003, ApJ, 591, 1220

Lutz, D. 1999, in The Universe as seen by ISO, ed. P. Cox, \& M. F. Kessler, ESA SP-427 (Noordwijk: ESA), 623

Lutz, D., Feuchtgruber, H., Genzel, R., et al. 1996, A\&A, 315, L269

Mathis, J. S. 1996, ApJ, 472, 643

Mathis, J. S., \& Whiffen, G. 1989, ApJ, 341, 808

Moore, T. J. T., Lumsden, S. L., Ridge, N. A., \& Puxley, P. J. 2005, MNRAS, 359, 589

O’Donnell, J. E. 1994, ApJ, 422, 158

Ossenkopf, V. 1991, A\&A, 251, 210

Ossenkopf, V., \& Henning, Th. 1994, A\&A, 291, 943

Posch, Th., Mutschke, H., \& Andersen, A. 2004, ApJ, 616, 1167

Przygodda, F., van Boekel, R., Ábraham, P., et al. 2003, A\&A, 412, L43

Quinten, M., Kreibig, U., Henning, Th., \& Mutschke, H. 2002, Appl. Opt., 41, 7102

Rouleau, F., \& Martin, P. G. 1991, ApJ, 377, 526

Savage, B. D., \& Sembach, K. R. 1996, ARA\&A, 34, 279
Schulz, N. S., Cui, W., Canizares, C. R., et al. 2002, ApJ, 565, 1141

Serkowski, K., Mathewson, D. S., \& Ford, V. L. 1975, ApJ, 196, 261

Snow, T. P., \& Witt, A. N. 1996, ApJ, 468, L65

Sofia, U. J., \& Meyer, D. M. 2001, ApJ, 554, L221 (Erratum, 558, L147)

Stognienko, R., Henning, Th., \& Ossenkopf, V. 1995, A\&A, 296, 797

Takeushi, T., Clarke, C. J., \& Lin, D. N. C. 2005, ApJ, 627, 286

Telesco, C. M., \& Knacke, R. F. 1991, ApJ, 372, L29

van Boekel, R., Waters, L. B. F. M., Dominik, C., et al. 2003, A\&A, 400, L21

van Boekel, R., Min, M., Waters, L. B. F. M., et al. 2005, A\&A, 437, 189

Voshchinnikov, N. V. 2004, Astrophys. \& Space Phys. Rev., 12, 1

Voshchinnikov, N. V., \& Il'in, V. B. 1987, SvAL, 13, 157

Voshchinnikov, N. V., \& Krügel, E. 1999, A\&A, 352, 508

Voshchinnikov, N. V., \& Mathis, J. S. 1999, ApJ, 526, 257

Voshchinnikov, N. V., Il'in, V. B., \& Henning, Th. 2005, A\&A, 429, 371

Wegner, W. 2002, BaltA, 11, 1

Wright, E. L. 1987, ApJ, 320, 818

Wolff, M., Clayton, G. C., Martin, P. G., \& Schulte-Ladbeck, R. E. 1994, ApJ, 423, 412

Wolff, M., Clayton, G. C., \& Gibson, S. J. 1998, ApJ, 503, 815

Zubko, V. G., Krełowski, J., \& Wegner, W. 1996, MNRAS, 283, 577 